\title{
PULMONARY EMBOLISM BY SMALL BLOOD CLOTS PHYSIOLOGICAL RESPONSES IN THE ANAESTHETIZED DOG
}

\author{
BY
}

\author{
ROBERT MARSHALL AND P. R. ALLISON
}

\author{
From the Nuffield Department of Surgery, Radcliffe Infirmary, Oxford
}

Embolization of the pulmonary arteries of anaesthetized dogs by large blood clots or thrombi usually causes no disturbance of heart rhythm or of respiration at the time of impaction of the embolus (Allison, Dunnill, and Marshall, 1960 ; Marshall, Allison, Sabiston, Bosman, and Dunnill, in preparation) and considerable recovery of lung function occurs during the first two weeks after the embolization. It is possible that human pulmonary embolism of severe degree is due to the impaction of a large embolus after multiple small emboli have caused an initial partial obstruction of the pulmonary circulation. Experimental work in animals also suggests that embolism of small arteries, rather than large arteries, initiates reflexes which may increase the pulmonary vascular resistance and cause even more obstruction to the circulation.

The present investigation was carried out to observe the respiratory and haemodynamic effects of repeated embolism by small blood clots and the recovery of function after the embolization.

\section{METHODS}

The material used for embolization in these experiments was fresh blood clot. The dogs were premedicated with morphine, $1.1 \mathrm{mg} / \mathrm{kg}$., and chlorpromazine, $1.0 \mathrm{mg} . / \mathrm{kg}$., and anaesthetized with pentobarbitone. The dogs were bled into sterile beakers, the blood for each injection being in a separate beaker. The blood was allowed to stand until it had formed a firm clot which was then chopped with sterile scissors, poured into a syringe, and injected slowly intravenously. In some experiments the blood was injected into the foreleg vein through a thinwalled 18 gauge needle and in others it was injected through a polythene tube inserted by way of the femoral vein into the inferior vena cava. The internal diameter of both needle and tube was $1 \mathrm{~mm}$.

Before the clots were injected the functional residual capacity (F.R.C.) and diffusing capacity of the lungs were measured (Marshall et al., in preparation) and cardiac catheterization was carried out to measure the pulmonary arterial pressure. In most of the dogs the catheter was left in the main pulmonary artery during the injection of the clots. Cannulae were inserted into the left femoral artery for recording pressure, into the right femoral artery for withdrawing blood, into the superior vena cava for measuring central venous pressure, and into the right femoral vein for the injection of dye and blood clots. A cuffed endotracheal tube was inserted and respiration was recorded using a spirometer and kymograph. In most of the experiments the dogs breathed air enriched with oxygen but in the experiments where the right-to-left shunt was measured (see below) the spirometer contained $100 \%$ oxygen and the dog's lungs were washed out with oxygen before connexion to the spirometer. An intra-oesophageal balloon connected to a capacitance manometer was used to measure intrathoracic pressure, and the output from this manometer, together with a volume tracing obtained from a low torque potentiometer attached to the spirometer, were recorded from a cathode ray oscilloscope. In four of the dogs dye curves were recorded before the injection of the clots and at the height of the pulmonary artery pressure response. A constant quantity of the dye (Coomassie blue) was injected into the inferior vena cava and the blood from the femoral artery was withdrawn through a Colson densitometer at a constant rate of $30 \mathrm{ml}$. per minute. The cardiac output was measured by the Fick method at the beginning and end of the experiment immediately before dye curves. The dye curves obtained during the experiment were used to determine changes in cardiac output relative to the calibrated curves.

In some dogs simultaneous samples of arterial blood and end-tidal gas were taken before the injection of clot, at the height of the pulmonary artery pressure rise, and again about ten minutes after the clot was injected. The end-tidal samples were taken from the lower end of the intratracheal tube about half way down the trachea, and cross wires were arranged to give a turbulent flow of gas at the sampling point. The oxygen tension of the gas samples and of the arterial blood was measured using a Clark electrode. An attempt was also made to measure the end-tidal, arterial $\mathrm{PCO}_{2}$ difference in these samples (Severinghaus, Swenson, Finley, Lategola, and Williams, 1961). 
but the method of measuring arterial $\mathrm{PCO}_{2}$ (from $p \mathrm{H}$ and $\mathrm{CO}_{2}$ content) proved to be too inaccurate. An estimate of the end-tidal, arterial $\mathrm{PCO}_{2}$ difference was made at the beginning and end of each experiment by calculating arterial $\mathrm{PCO}_{2}$ from the mixed venous $\mathrm{PcO}_{2}$ obtained by a rebreathing method (Hackney, Sears, and Collier, 1958).

From the tracings of intra-oesophageal pressure and tidal volume the dynamic compliance of the lungs was measured by the method of Neergaard and Wirz (1927). The non-elastic resistance of the lungs was obtained by drawing a pressure-volume loop from the continuous tracings of intra-oesophageal pressure and tidal volume. A mean value for non-elastic resistance was calculated from the equation $R=\frac{A\left(t_{i} \times t_{e}\right)}{V^{2}\left(t_{i}+t_{e}\right)}$ where $\mathbf{R}$ is the non-elastic resistance, $\mathbf{A}$ is the area enclosed by the pressure-volume loop, $\mathbf{V}$ is the tidal volume, and $t_{i}$ and $t_{e}$ are the times for inspiration and expiration respectively. The resistance of the endotracheal and connecting tubes was $4.0 \mathrm{~cm}$. water $/ 1$. $/ \mathrm{sec}$. at the flow rates encountered in these dogs. This resistance has been subtracted from the measured resistance to give the values in Table VI.

The quantity of blood clot injected each time was usually $20 \mathrm{ml}$., and the injection was repeated three or four times within 30 to 60 minutes. In the later experiments of the series the dog was re-investigated one week after the first injection of clots. In some instances more clots were injected until the dog died; in other instances no clots were injected on this occasion but pulmonary function tests were carried out, the pulmonary artery pressure was measured, and the dog was killed by perfusion with formalin.

\section{Results}

EFFECT ON RESPIRATION.-The usual result of embolism by these small blood clots was rapid, shallow respiration, but the effect was very variable and in some instances there was a transient increase in the rate of breathing with no change in tidal volume (Table I). The changes in the rate and depth of respiration usually started a little later than the increase in pulmonary artery pressure and in some cases did not occur until the pulmonary artery pressure had started to fall. When clots of equal volume were given at intervals of a few minutes the changes in respiration were often more marked with the first clot than they were with later clots. The final injection of clot which caused death usually caused rapid, shallow breathing soon terminating in apnoea, even though earlier injections may have had no effect on respiration.

Any changes in F.R.C. which occurred following impaction of the emboli were probably small. From the spirometer tracing changes in F.R.C. cannot with certainty be distinguished from changes in oxygen uptake. In some of the dogs the slope of the tracing was the same before and after the embolism with a sudden change in level at the time of impaction. Such changes were usually small and of the order of $20 \mathrm{ml}$. increase in F.R.C. With two exceptions (dogs 1 and 8) the F.R.C. measured on different days changed by less

TABLE I

THE EFFECT OF EMBOLISM BY SMALL BLOOD CLOTS ON RESPIRATORY RATE AND DEPTH

\begin{tabular}{|c|c|c|c|c|c|c|c|c|c|c|c|c|c|}
\hline \multirow[t]{2}{*}{$\begin{array}{l}\text { Dog } \\
\text { No. }\end{array}$} & \multirow[t]{2}{*}{ Date } & \multicolumn{4}{|c|}{$\begin{array}{l}\text { Minimum Tidal Volume } \\
\text { (\% of Initial) } \\
\text { Embolus No. }\end{array}$} & \multicolumn{4}{|c|}{$\begin{array}{l}\text { Maximum Respiratory } \\
\text { Rate (\% of Initial) } \\
\text { Embolus No. }\end{array}$} & \multicolumn{4}{|c|}{$\begin{array}{l}\text { Time for Rate or Tidal Volume to } \\
\text { Return to } 90 \% \text { of Recovery Level } \\
\text { (sec.) }\end{array}$} \\
\hline & & 1 & 2 & 3 & 4 & 1 & 2 & 3 & 4 & 1 & 2 & 3 & 4 \\
\hline 1 & $\begin{array}{r}16 / 5 \\
7 / 6 \\
17 / 6\end{array}$ & $\begin{array}{l}47 \\
31 \\
19\end{array}$ & $\begin{array}{l}50 \\
82 \\
72\end{array}$ & $\begin{array}{l}57 \\
69 \\
82\end{array}$ & & $\begin{array}{l}357 \\
600 \\
600\end{array}$ & $\begin{array}{l}250 \\
185 \\
240\end{array}$ & $\begin{array}{l}235 \\
143 \\
170\end{array}$ & & $\begin{array}{r}75 \\
95 \\
220\end{array}$ & $\begin{array}{r}180 \\
52 \\
200\end{array}$ & $\begin{array}{r}65 \\
62 \\
130\end{array}$ & \\
\hline 2 & $\begin{array}{r}16 / 5 \\
7 / 6 \\
17 / 6\end{array}$ & $\begin{array}{l}30 \\
50 \\
14\end{array}$ & $\begin{array}{l}77 \\
\mathbf{U}\end{array}$ & $\begin{array}{l}88 \\
\mathbf{U}\end{array}$ & & $\begin{array}{l}150 \\
138 \\
165\end{array}$ & $\begin{array}{r}180 \\
\mathbf{U} \\
200\end{array}$ & $\begin{array}{r}140 \\
\mathbf{U} \\
400\end{array}$ & & $\begin{array}{r}78 \\
120 \\
75\end{array}$ & $\begin{array}{l}105 \\
105\end{array}$ & $\begin{array}{c}25 \\
\text { Died }\end{array}$ & \\
\hline 3 & $\begin{array}{l}13 / 6 \\
20 / 6\end{array}$ & $\begin{array}{l}78 \\
84\end{array}$ & $\underset{55}{U}$ & $\mathbf{U}$ & $\mathbf{U}$ & $\begin{array}{l}200 \\
180\end{array}$ & $\underset{300}{U}$ & $\underset{300}{\mathbf{U}}$ & $\mathbf{U}$ & $\begin{array}{r}120 \\
40\end{array}$ & 170 & Died & \\
\hline 4 & $\begin{array}{r}27 / 6 \\
6 / 7\end{array}$ & $\begin{array}{l}32 \\
43\end{array}$ & $\begin{array}{l}38 \\
23\end{array}$ & 33 & & $\begin{array}{l}300 \\
250\end{array}$ & $\begin{array}{l}250 \\
200\end{array}$ & 300 & & 133 & $\begin{array}{l}110 \\
\text { Died }\end{array}$ & 145 & \\
\hline 5 & $\begin{array}{r}11 / 7 \\
18 / 7 \\
2 / 8\end{array}$ & $\begin{array}{r}45 \\
41 \\
\text { U }\end{array}$ & $\begin{array}{l}\text { U } \\
75 \\
79\end{array}$ & $\begin{array}{l}\text { U } \\
\text { Died }\end{array}$ & Apnoea & $\begin{array}{r}380 \\
\mathbf{U}\end{array}$ & $\begin{array}{l}U \\
125 \\
220\end{array}$ & $\begin{array}{l}U \\
220 \\
180\end{array}$ & 170 & $\begin{array}{r}60 \\
160\end{array}$ & $\begin{array}{l}41 \\
60\end{array}$ & $\begin{array}{c}95 \\
\text { Died }\end{array}$ & 250 \\
\hline 6 & $\begin{array}{l}14 / 7 \\
19 / 7\end{array}$ & $\underset{\mathbf{U}}{\mathbf{U}}$ & $\mathbf{U}$ & $\mathbf{U}$ & Apnoea & $\begin{array}{l}167 \\
200\end{array}$ & $\begin{array}{l}167 \\
142\end{array}$ & $\begin{array}{l}140 \\
200\end{array}$ & 150 & $\begin{array}{l}150 \\
200\end{array}$ & $\begin{array}{l}100 \\
100\end{array}$ & 120 & Died \\
\hline 7 & $\begin{array}{r}9 / 8 \\
16 / 8\end{array}$ & $\begin{array}{l}70 \\
\mathbf{U}\end{array}$ & $\begin{array}{l}\mathbf{U} \\
\mathbf{8 1}\end{array}$ & $\begin{array}{l}\text { Died } \\
\text { Dis }\end{array}$ & 89 & $\begin{array}{l}300 \\
150\end{array}$ & $\begin{array}{l}114 \\
240\end{array}$ & $\begin{array}{l}200 \\
300\end{array}$ & 190 & 150 & 75 & Died & 40 \\
\hline 8 & $25 / 8$ & $\mathbf{U}$ & $\mathbf{U}$ & $\mathbf{U}$ & & 200 & $\mathbf{U}$ & 170 & & 60 & & & \\
\hline 9 & $11 / 10$ & $\mathbf{U}$ & Died & & & 160 & 300 & & & 60 & Died & & \\
\hline 10 & $24 / 10$ & $\mathbf{U}$ & $\mathbf{U}$ & $\mathbf{U}$ & & $\mathbf{U}$ & $\mathbf{U}$ & $\mathbf{U}$ & & & & & \\
\hline
\end{tabular}


than $10 \%$ (Table IV). In dog 1 there was an increase of $16 \%$ and in dog 8 a decrease of $18 \%$.

CaRDIAC EFFECTS.-Injection of $20 \mathrm{ml}$. of blood clot often produced no change in the electrocardiogram. When the clot did cause a large rise in pulmonary artery pressure and a fall in systemic pressure the heart rate increased, and in some cases, particularly after several injections of clot, the limb leads showed deepening of the $S$ wave and inversion of the $T$ wave. The heart continued to beat for several minutes after the onset of terminal apnoea.
The Pulmonary Vascular Pressures and RESistance.-The effect of pulmonary embolism on the pulmonary vascular pressures in six dogs is shown in Table II. Embolism by $20 \mathrm{ml}$. of blood clot caused a rise in pulmonary artery pressure in almost all instances, but the rise was variable and was particularly small in dogs 7 and 10. The increase in pulmonary artery pressure was often very considerable in dogs which showed no change in rate or depth of respiration. The pulmonary artery pressure rose to its maximum about 20 to 30 seconds after the completion of the

TABLE II

THE EFFECT OF EMBOLISM BY SMALL BLOOD CLOTS ON THE PULMONARY ARTERY PRESSURE

Pulmonary Artery Pressure (mm. Hg)

\begin{tabular}{|c|c|c|c|c|c|c|c|c|c|c|}
\hline \multirow{3}{*}{$\begin{array}{l}\text { Dog } \\
\text { No. }\end{array}$} & \multirow{3}{*}{ Date } & \multicolumn{8}{|c|}{ - } & \multirow{3}{*}{ Later } \\
\hline & & \multicolumn{2}{|c|}{ First Embolus } & \multicolumn{2}{|c|}{ Second Embolus } & \multicolumn{2}{|c|}{ Third Embolus } & \multicolumn{2}{|c|}{ Fourth Embolus } & \\
\hline & & Before & After & Before & After & Before & After & Before & $\overrightarrow{\text { After }}$ & \\
\hline 3 & $13 / 6$ & $32 / 11$ & $39 ; 23$ & $35 / 21$ & $41 / 28$ & $29 / 21$ & $35 / 25$ & $31 / 23$ & $36 / 30$ & \\
\hline 4 & $\begin{array}{r}27 ' 6 \\
6 / 7\end{array}$ & $\begin{array}{l}16 / 7 \\
6 /-1\end{array}$ & $\begin{array}{l}28 / 18 \\
26 / 13\end{array}$ & $\begin{array}{l}21 / 12 \\
17 / 5\end{array}$ & $\begin{array}{l}37 / 23 \\
42 / 18\end{array}$ & $32 / 21$ & $46 / 28$ & & & $40: 23$ \\
\hline 5 & $\begin{array}{r}11 / 7 \\
18 / 7 \\
2 / 8\end{array}$ & $\begin{array}{r}7 / 2 \\
5 /-3 \\
16 / 5\end{array}$ & $\begin{array}{l}33 / 14 \\
17 / 5 \\
51 / 24\end{array}$ & $\begin{array}{l}16 / 18 \\
15 / 12 \\
24 / 12\end{array}$ & $\begin{array}{c}37 / 18 \\
35 / 15 \\
139 / 40\end{array}$ & $\begin{array}{c}27 / 17 \\
22^{\prime} 12 \\
36 / 24\end{array}$ & $\begin{array}{r}48 / 25 \\
53^{\prime} 26 \\
150,43\end{array}$ & & & $\begin{array}{l}28 / 17 \\
27 / 13\end{array}$ \\
\hline 6 & $\begin{array}{l}14 / 7 \\
19 / 7\end{array}$ & $\begin{array}{l}13 / 0 \\
18 / 0\end{array}$ & $\begin{array}{l}65 / 37 \\
79 / 46\end{array}$ & $\begin{array}{l}44 / 31 \\
38 / 26\end{array}$ & $\begin{array}{l}53 / 36 \\
71 / 46\end{array}$ & $\begin{array}{l}49 / 36 \\
53 / 35\end{array}$ & $\begin{array}{r}60 / 39 \\
151 / 79\end{array}$ & $129 / 74$ & $170 / 88$ & $40 / 36$ \\
\hline 7 & $\begin{array}{r}9 / 8 \\
16 / 8\end{array}$ & $\begin{array}{l}2^{\prime}-2 \\
12 / 0\end{array}$ & $\begin{array}{l}12 / 5 \\
43 / 24\end{array}$ & $\begin{array}{c}6 / 5 \\
23 / 16\end{array}$ & $\begin{array}{r}4 / 2 \\
94 / 39\end{array}$ & $\begin{array}{c}4^{\prime} 2 \\
40 / 26\end{array}$ & $\begin{array}{c}5 / 1 \\
100 / 39\end{array}$ & $4 / 1$ & $10 /-1$ & $5 / 1$ \\
\hline 8 & $25 / 8$ & $\begin{array}{l}18 / 7 \\
30 / 8\end{array}$ & $\begin{array}{l}46^{\prime} 26 \\
20^{\prime} 0\end{array}$ & $22 / 7$ & $43 / 20$ & $28 / 11$ & $56 / 26$ & & & \\
\hline 9 & $11 / 10$ & $19 / 0$ & $129 / 41$ & $57 / 14$ & $163 / 49$ & & & & & \\
\hline 10 & $\begin{array}{l}24 / 10 \\
31 / 10\end{array}$ & $\begin{array}{l}14 / 3 \\
18 / 2\end{array}$ & $17 / 6$ & $14 / 4$ & $18: 8$ & $15 / 5$ & 217 & & & \\
\hline
\end{tabular}

TABLE III

THE EFFECT OF EMBOLISM BY SMALL BLOOD CLOTS ON THE PULMONARY VASCULAR RESISTANCE

\begin{tabular}{|c|c|c|c|c|c|c|c|c|}
\hline & \multicolumn{2}{|c|}{ First Embolus } & \multicolumn{2}{|c|}{ Second Embolus } & \multicolumn{2}{|c|}{ Third Embolus } & \multirow{2}{*}{ Later } & \multirow{2}{*}{$\begin{array}{l}\text { One } \\
\text { Week } \\
\text { Later }\end{array}$} \\
\hline & Before & $\overline{\text { After }}$ & Before & $\overline{\text { After }}$ & Before & $\overline{\text { After }}$ & & \\
\hline \multirow{3}{*}{$\begin{array}{l}\text { Dog No. } 7(16 / 8) \\
\text { Cardiac output }(1 . / \mathrm{m} .) \\
\text { P.A. pressure* (mm. Hg) } \\
\text { Mean P.A. pressure } \\
\text { (mm. Hg) } \\
\text { Resistance† } \\
\text { (mm. Hg/l./min.) }\end{array}$} & $\begin{array}{l}1 \cdot 17 \\
12 / 0\end{array}$ & $\begin{array}{r}1 \cdot 26 \\
36 / 21\end{array}$ & $\begin{array}{l}1 \cdot 15 \\
23 ! 16\end{array}$ & $\begin{array}{r}1 \cdot 13 \\
59 / 37\end{array}$ & & & & \\
\hline & 9 & 31 & 23 & 49 & & & & \\
\hline & $7 \cdot 7$ & $24 \cdot 6$ & $20 \cdot 0$ & $43 \cdot 4$ & & & & \\
\hline \multirow{3}{*}{$\begin{array}{l}\text { Dog No. } 8(25 / 8) \\
\text { Cardiac output (1./m.) } \\
\text { P.A. pressure (mm. Hg) } \\
\text { Mean P.A. pressure } \\
\text { (mm. Hg) } \\
\text { Resistance } \\
\text { (mm. Hg/l./min.) }\end{array}$} & $\begin{array}{l}2 \cdot 27 \\
18 / 7\end{array}$ & $\begin{array}{l}2 \cdot 20 \\
46 / 26\end{array}$ & $22 / 7$ & $\begin{array}{l}2 \cdot 18 \\
43 / 20\end{array}$ & $\begin{array}{l}2 \cdot 58 \\
28 / 11\end{array}$ & $\begin{array}{l}2 \cdot 69 \\
56 / 26\end{array}$ & $\begin{array}{l}2 \cdot 49 \\
38 / 15\end{array}$ & $\begin{array}{l}1.96 \\
2010\end{array}$ \\
\hline & 16 & 38 & 17 & 33 & 22 & 41 & 28 & 12 \\
\hline & $7 \cdot 0$ & $17 \cdot 3$ & & $15 \cdot 2$ & $8 \cdot 5$ & $15 \cdot 3$ & $11 \cdot 2$ & $6 \cdot 1$ \\
\hline \multirow{3}{*}{$\begin{array}{l}\text { Dog No. } 9(11 / 10) \\
\text { Cardiac output (1./m.) } \\
\text { P.A. pressure (mm. Hg) } \\
\text { Mean P.A. pressure } \\
\text { (mm. Hg) } \\
\text { Resistance } \\
\text { (mm. Hg } / 1 . / \mathrm{min} .)\end{array}$} & $\begin{array}{l}0.92 \\
19 / 0\end{array}$ & $\begin{array}{c}0 \cdot 73 \\
129 / 41\end{array}$ & $\begin{array}{l}1 \cdot 12 \\
57 / 14\end{array}$ & & & & & \\
\hline & 11 & 75 & 33 & & & & & \\
\hline & $12 \cdot 0$ & 103 & 29.5 & & & & & \\
\hline \multirow{3}{*}{$\begin{array}{l}\text { Dog No. } 10(25 / 10) \\
\text { Cardiac output (1./m.) } \\
\text { P.A. pressure (mm. Hg) } \\
\text { Mean P.A. pressure } \\
\text { (mm. Hg) } \\
\text { Resistance } \\
\text { (mm. Hg/l./min.) }\end{array}$} & $\begin{array}{l}1.80 \\
14^{\prime} 3\end{array}$ & $\begin{array}{l}1.20 \\
17 ! 6\end{array}$ & $\begin{array}{l}1.94 \\
14 / 4\end{array}$ & $\begin{array}{l}1 \cdot 52 \\
18 ' 8\end{array}$ & $\begin{array}{l}1.80 \\
15.5\end{array}$ & $\begin{array}{l}1 \cdot 88 \\
21 / 7\end{array}$ & & $\begin{array}{l}1 \cdot 70 \\
18 / 2\end{array}$ \\
\hline & 12 & 15 & 12 & 16 & 13 & 17 & & 12 \\
\hline & 6.7 & $12 \cdot 5$ & $6 \cdot 2$ & 10.5 & $7 \cdot 2$ & 9.0 & & $7 \cdot 1$ \\
\hline
\end{tabular}

* The reference level for pulmonary artery pressure was the front of the sternum.

+ For the calculation of resistance the zero for pulmonary artery mean pressure is taken as $5 \mathrm{~cm}$. below the front of the sternum; the left atrial pressure has been neglected. 
injection and then fell gradually, reaching a steady level, usually higher than the initial level, after about one to two minutes. In the six dogs in which measurements were repeated after one week the pressure had fallen to within normal limits and was usually only a few millimetres of mercury above the initial level. The injection of further clots in four of these dogs showed that the capacity of the pulmonary arteries was reduced by the clot ; after one week the same dose of clot as was given originally caused a much greater increase in pulmonary artery pressure and usually resulted in death after two or three injections. The rise in pulmonary artery pressure after the first injection was much greater in dog 9 than in the other dogs, and the second injection was fatal. This dog was smaller than the other dogs $(10.6 \mathrm{~kg}$., whilst the mean weight for the group of eight dogs was $16.1 \mathrm{~kg}$.) but it was also anaemic (oxygen capacity 10.8 volumes \%) and necropsy showed evidence of chronic pulmonary infection.

The systemic pressure usually showed only a small fall with each embolus. In $\operatorname{dog} 6$ the pressures were low throughout most of the period of measurement on both days. The central venous pressure usually showed only small changes with each embolus until the final fatal dose when large $\mathrm{V}$ waves were present and the mean pressure rose to $12 \mathrm{~mm}$. $\mathrm{Hg}$ above the sternal angle.

The pulmonary vascular resistance was measured in four dogs (Table III). The changes in cardiac output as a result of the embolization were small. The resistance was high just after the embolus when the pulmonary artery pressure was high, but it fell in the following few minutes.

Evidence of Right-to-Left Shunt of BloOd. - Simultaneous measurements of end-tidal $\mathrm{Po}_{2}$ and arterial $\mathrm{Po}_{2}$ were made before and after embolization in five of the dogs breathing almost $100 \%$ oxygen. The right-to-left shunt calculated

TABLE IV

THE EFFECT OF EMBOLISM BY SMALL BLOOD CLOTS ON THE PULMONARY DIFFUSING CAPACITY FOR CARBON MONOXIDE

\begin{tabular}{|c|c|c|c|c|}
\hline \multirow{2}{*}{$\begin{array}{l}\text { Dog } \\
\text { No. }\end{array}$} & \multirow{2}{*}{ Date } & \multicolumn{2}{|c|}{$\begin{array}{l}\text { Diffusing Capacity for CO } \\
(\mathrm{ml} / \mathrm{min} . / \mathrm{mm} . \mathrm{Hg})\end{array}$} & \multirow{2}{*}{ (mi.) } \\
\hline & & $\begin{array}{c}\text { Before } \\
\text { Embolus }\end{array}$ & $\begin{array}{c}\text { After } \\
\text { Embolus }\end{array}$ & \\
\hline 1 & $\begin{array}{r}14 / 4 \\
16 / 5 \\
7 / 6 \\
17 / 6 \\
27 / 6 \\
4 / 7 \\
18 / 7\end{array}$ & $\begin{array}{l}7.84 \\
7.08 \\
7.06 \\
6.61 \\
5.50 \\
4.61 \\
6.30\end{array}$ & $\begin{array}{l}6 \cdot 53 \\
6 \cdot 68 \\
3 \cdot 70\end{array}$ & $\begin{array}{l}514 \\
519 \\
530 \\
597 \\
596 \\
544 \\
590\end{array}$ \\
\hline 2 & $\begin{array}{r}16 / 5 \\
7 / 6 \\
17 / 6\end{array}$ & $\begin{array}{l}6.87 \\
5.02 \\
5.62\end{array}$ & $\begin{array}{l}5 \cdot 37 \\
4 \cdot 07\end{array}$ & $\begin{array}{l}418 \\
406 \\
398\end{array}$ \\
\hline 3 & $\begin{array}{l}13 / 6 \\
20 / 6\end{array}$ & $\begin{array}{l}6 \cdot 79 \\
6 \cdot 18\end{array}$ & $5 \cdot 21$ & $\begin{array}{l}610 \\
610\end{array}$ \\
\hline 4 & $\begin{array}{r}27 / 6 \\
6: 7\end{array}$ & $\begin{array}{l}5 \cdot 65 \\
6 \cdot 04\end{array}$ & $5 \cdot 78$ & $\begin{array}{l}418 \\
444\end{array}$ \\
\hline 5 & $\begin{array}{r}11 / 7 \\
18 / 7 \\
2 / 8\end{array}$ & $\begin{array}{l}5.97 \\
5 \cdot 20 \\
4.80\end{array}$ & $\begin{array}{l}4 \cdot 08 \\
2 \cdot 27\end{array}$ & $\begin{array}{l}531 \\
545 \\
572\end{array}$ \\
\hline 6 & $\begin{array}{l}14 / 7 \\
19 / 7\end{array}$ & $\begin{array}{l}7 \cdot 11 \\
5 \cdot 35\end{array}$ & 3.03 & $\begin{array}{l}766 \\
808\end{array}$ \\
\hline 7 & $\begin{array}{r}9 / 8 \\
16 / 8\end{array}$ & $\begin{array}{l}7 \cdot 83 \\
6 \cdot 78\end{array}$ & 4.42 & $\begin{array}{l}611 \\
572\end{array}$ \\
\hline 8 & $\begin{array}{l}25 / 8 \\
30 / 8\end{array}$ & $\begin{array}{l}8 \cdot 34 \\
7 \cdot 12\end{array}$ & 7.57 & $\begin{array}{l}720 \\
590\end{array}$ \\
\hline 10 & $\begin{array}{l}24 / 10 \\
30 ! 10\end{array}$ & $\begin{array}{l}9.99 \\
8.85\end{array}$ & 8.40 & $\begin{array}{l}729 \\
768\end{array}$ \\
\hline
\end{tabular}

TABLE V

THE EFFECT OF EMBOLISM BY SMALL BLOOD CLOTS ON THE COMPLIANCE OF THE LUNGS

Compliance (ml.'cm. water)

\begin{tabular}{|c|c|c|c|c|c|c|c|c|c|c|}
\hline \multirow{3}{*}{$\begin{array}{l}\text { Dog } \\
\text { No. }\end{array}$} & \multirow{3}{*}{ Date } & \\
\hline & & \multicolumn{2}{|c|}{ First Embolus } & \multicolumn{2}{|c|}{ Second Embolus } & \multicolumn{2}{|c|}{ Third Embolus } & \multicolumn{2}{|c|}{ Fourth Embolus } & \multirow{2}{*}{ Later } \\
\hline & & Before & After & Before & After & Before & After & Before & After & \\
\hline 1 & $\begin{array}{r}7 / 6 \\
17 / 6 \\
18 / 7\end{array}$ & $\begin{array}{l}20 \\
23 \\
16\end{array}$ & $\begin{array}{l}38 \\
42 \\
29\end{array}$ & $\begin{array}{l}23 \\
27\end{array}$ & $\begin{array}{l}29 \\
36\end{array}$ & $\begin{array}{l}25 \\
26\end{array}$ & $\begin{array}{l}29 \\
34\end{array}$ & & & $\begin{array}{l}22 \\
26\end{array}$ \\
\hline 2 & $\begin{array}{r}16: 5 \\
7 / 6 \\
17 / 6\end{array}$ & $\begin{array}{l}35 \\
36 \\
30\end{array}$ & $\begin{array}{l}45 \\
56 \\
44\end{array}$ & $\begin{array}{l}46 \\
30\end{array}$ & $\begin{array}{l}46 \\
46\end{array}$ & $\begin{array}{l}39 \\
35\end{array}$ & $\begin{array}{l}42 \\
51\end{array}$ & & & \\
\hline 3 & $\begin{array}{l}13 / 6 \\
20 / 6\end{array}$ & 18 & 18 & $\begin{array}{l}20 \\
22\end{array}$ & $\begin{array}{l}23 \\
30\end{array}$ & $\begin{array}{l}15 \\
17\end{array}$ & $\begin{array}{l}15 \\
30\end{array}$ & 15 & 15 & \\
\hline 4 & $\begin{array}{r}27 / 6 \\
6: 7\end{array}$ & $\begin{array}{l}34 \\
33\end{array}$ & $\begin{array}{l}64 \\
46\end{array}$ & $\begin{array}{l}28 \\
34\end{array}$ & $\begin{array}{l}34 \\
27\end{array}$ & $\begin{array}{l}30 \\
27\end{array}$ & $\begin{array}{l}44 \\
53\end{array}$ & & & 30 \\
\hline 5 & $\begin{array}{r}11 / 7 \\
18 / 7 \\
28\end{array}$ & $\begin{array}{l}32 \\
21 \\
29\end{array}$ & $\begin{array}{l}57 \\
40 \\
29\end{array}$ & $\begin{array}{l}53 \\
34 \\
29\end{array}$ & $\begin{array}{l}57 \\
40 \\
32\end{array}$ & $\begin{array}{l}57 \\
38 \\
30\end{array}$ & $\begin{array}{l}61 \\
38 \\
45\end{array}$ & 34 & 80 & $\begin{array}{l}50 \\
30\end{array}$ \\
\hline 6 & $\begin{array}{l}14: 7 \\
19: 7\end{array}$ & $\begin{array}{r}12 \\
8\end{array}$ & $\begin{array}{l}28 \\
15\end{array}$ & $\begin{array}{l}28 \\
13\end{array}$ & $\begin{array}{l}28 \\
12\end{array}$ & $\begin{array}{l}21 \\
10\end{array}$ & $\begin{array}{l}23 \\
16\end{array}$ & 14 & 20 & 17 \\
\hline 7 & $\begin{array}{r}9 / 8 \\
16.8\end{array}$ & $\begin{array}{l}25 \\
20\end{array}$ & $\begin{array}{l}43 \\
24\end{array}$ & $\begin{array}{l}36 \\
20\end{array}$ & $\begin{array}{l}36 \\
20\end{array}$ & 36 & 36 & 36 & 37 & 28 \\
\hline 8 & $25 / 8$ & 15 & 19 & 18 & 19 & 17 & 19 & & & 18 \\
\hline 9 & $11 / 10$ & 36 & 36 & 39 & 62 & & & & & \\
\hline 10 & $24 / 10$ & 14 & 16 & 15 & 15 & 15 & 16 & & & 16 \\
\hline
\end{tabular}




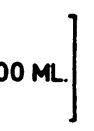

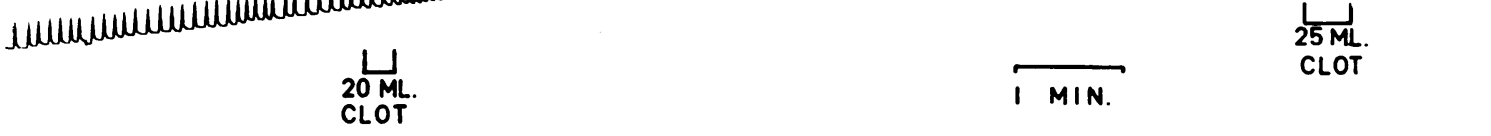

FIG. 1. Spirometer tracing showing the effect of injection of small blood clots intravenously. The second injection of clot caused fatal apnoea $(\operatorname{dog} 4,6 / 7)$.

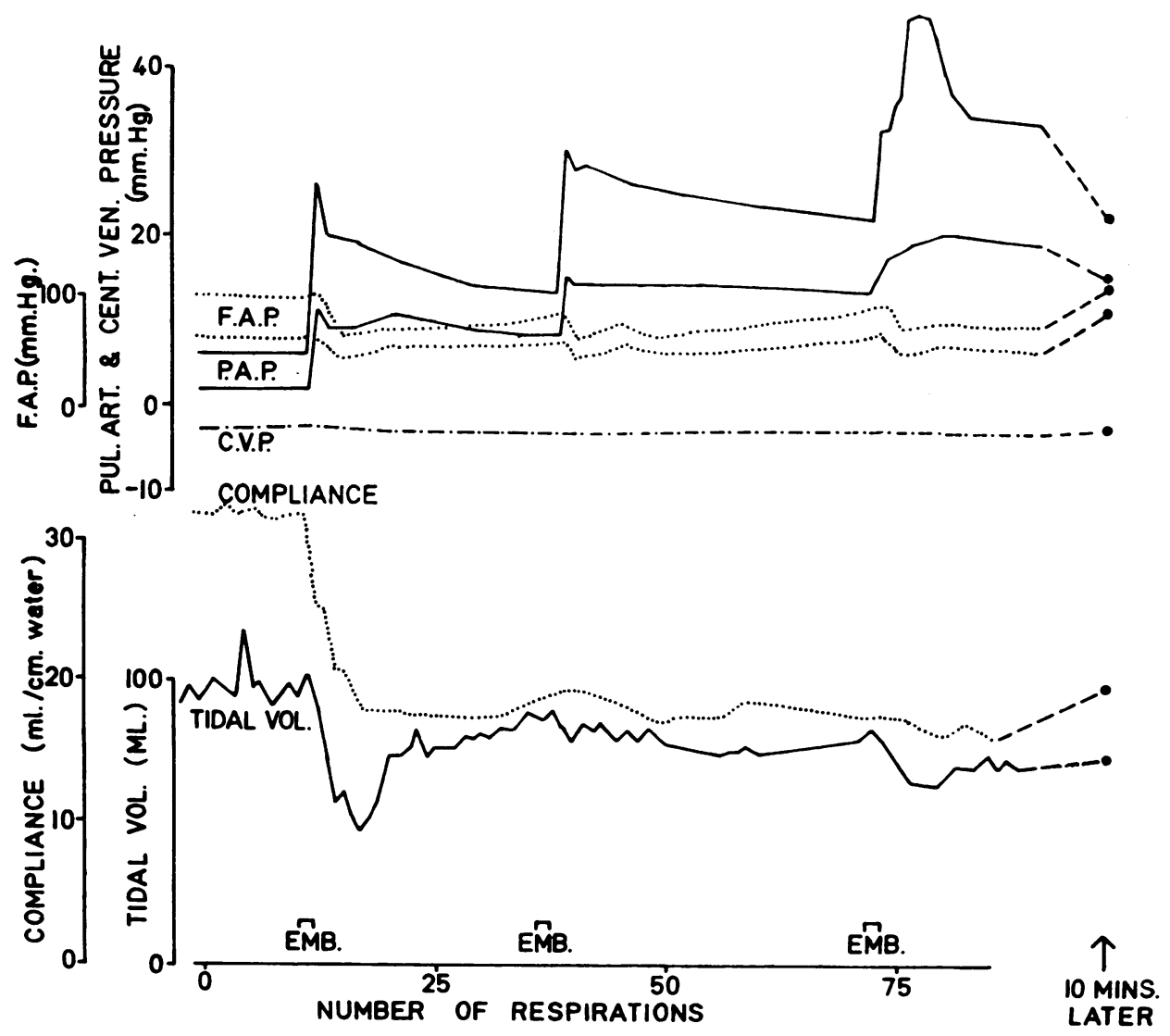

FIG. 2. The effect on respiration and haemodynamics of the intravenous injection of small blood clot (dog 5, 11/7): F.A.P. =femoral artery pressure; P.A.P.=pulmonary artery pressure; C.V.P. =central venous pressure; the time scale is shown as " number of respirations."

from the difference in oxygen tension between alveolar gas and arterial blood was usually less than $1 \%$ and was not affected by the embolization.

End-tidal, Arterial $\mathrm{PCO}_{2}$ Differences.-In seven of the dogs the end-tidal gas was sampled and immediately afterwards an alveolar sample equilibrated with mixed venous blood was obtained for estimation of arterial $\mathrm{PCO}_{2}$. These measurements were made before the embolization and repeated after all the emboli had been given. Only small, and probably insignificant, changes in end-tidal, arterial $\mathrm{PCO}_{2}$ difference were found. This result is similar to the findings after embolization by large thrombi (Marshall et al., in preparation). 
TABLE VI

THE EFFECT OF EMBOLISM BY SMALL BLOOD CLOTS ON THE NON-ELASTIC RESISTANCE OF THE LUNGS

(Non-elastic Resistance (cm. water/1./sec.)

\begin{tabular}{|c|c|c|c|c|c|c|c|c|c|c|}
\hline \multirow{3}{*}{$\begin{array}{l}\text { Dog } \\
\text { No. }\end{array}$} & \multirow{3}{*}{ Date } & \\
\hline & & \multicolumn{2}{|c|}{ First Embolus } & \multicolumn{2}{|c|}{ Second Embolus } & \multicolumn{2}{|c|}{ Third Embolus } & \multicolumn{2}{|c|}{ Fourth Embolus } & \multirow{2}{*}{ Later } \\
\hline & & Before & After & Before & After & Before & After & Before & After & \\
\hline 1 & $\begin{array}{r}7 / 6 \\
17 / 6 \\
18 / 7\end{array}$ & $\begin{array}{l}3 \cdot 33 \\
4 \cdot 54 \\
4 \cdot 10\end{array}$ & $\begin{array}{l}4 \cdot 15 \\
6.85 \\
4 \cdot 60\end{array}$ & $\begin{array}{l}4.04 \\
3.52\end{array}$ & $\begin{array}{l}3.82 \\
4.46\end{array}$ & $\begin{array}{l}4.02 \\
3.00\end{array}$ & $\begin{array}{l}3 \cdot 82 \\
3 \cdot 36\end{array}$ & & & $\begin{array}{l}4.36 \\
5.08\end{array}$ \\
\hline 2 & $\begin{array}{r}16 / 5 \\
2 / 6 \\
17 / 6\end{array}$ & $\begin{array}{l}4 \cdot 32 \\
5 \cdot 94 \\
4 \cdot 60\end{array}$ & $\begin{array}{r}4.92 \\
10 \cdot 30 \\
3.90\end{array}$ & $\begin{array}{l}6.67 \\
5 \cdot 57\end{array}$ & $\begin{array}{l}8.95 \\
6 \cdot 40\end{array}$ & $\begin{array}{l}5.82 \\
6.80\end{array}$ & $\begin{array}{l}6.90 \\
7.87\end{array}$ & & & \\
\hline 3 & $20 / 6$ & $2 \cdot 20$ & 3.02 & $2 \cdot 84$ & 3.06 & $4 \cdot 02$ & $10 \cdot 65$ & & & \\
\hline 4 & $\begin{array}{r}27: 6 \\
6 / 7\end{array}$ & $\begin{array}{l}4 \cdot 22 \\
3 \cdot 46\end{array}$ & $\begin{array}{l}6.42 \\
5.54\end{array}$ & $4 \cdot 25$ & $6 \cdot 23$ & $\begin{array}{l}5.45 \\
1.30\end{array}$ & $\begin{array}{l}6.11 \\
3.08\end{array}$ & & & \\
\hline 5 & $\begin{array}{r}11 / 7 \\
18 / 7 \\
2 / 8\end{array}$ & $\begin{array}{l}5.54 \\
0.87 \\
0.98\end{array}$ & $\begin{array}{l}7 \cdot 20 \\
4 \cdot 14 \\
1 \cdot 60\end{array}$ & $\begin{array}{l}6.05 \\
2.80\end{array}$ & $\begin{array}{l}6.50 \\
3.30 \\
1.60\end{array}$ & $\begin{array}{l}6.35 \\
1.98\end{array}$ & $\begin{array}{l}6 \cdot 13 \\
3 \cdot 08 \\
5 \cdot 30\end{array}$ & $1 \cdot 27$ & 5.09 & $2 \cdot 70$ \\
\hline 6 & $\begin{array}{l}14 / 7 \\
19 / 7\end{array}$ & $\begin{array}{l}4 \cdot 22 \\
2 \cdot 54\end{array}$ & $\begin{array}{r}12.80 \\
3.75\end{array}$ & & $\begin{array}{r}12.10 \\
3.73\end{array}$ & $\begin{array}{l}8.98 \\
2.00\end{array}$ & $\begin{array}{l}6.58 \\
3.55\end{array}$ & $3 \cdot 42$ & 4.60 & \\
\hline 7 & $\begin{array}{r}9^{\prime} 8 \\
16 / 8\end{array}$ & $\begin{array}{l}2 \cdot 85 \\
2 \cdot 51\end{array}$ & $\begin{array}{l}5.00 \\
2 \cdot 78\end{array}$ & $\begin{array}{l}4 \cdot 52 \\
2 \cdot 10\end{array}$ & $\begin{array}{l}3.64 \\
3.15\end{array}$ & $7 \cdot 39$ & 3.05 & $3 \cdot 42$ & $3 \cdot 85$ & \\
\hline 8 & $\begin{array}{l}25 / 8 \\
30 / 8\end{array}$ & $\begin{array}{l}3 \cdot 19 \\
3.99\end{array}$ & $3 \cdot 30$ & $2 \cdot 75$ & $3 \cdot 14$ & $2 \cdot 30$ & $3 \cdot 16$ & & & \\
\hline 9 & $11 / 10$ & $7 \cdot 48$ & $7 \cdot 37$ & $5 \cdot 10$ & $13 \cdot 70$ & & & & & \\
\hline 10 & $\begin{array}{l}24 / 10 \\
31 / 10\end{array}$ & $\begin{array}{l}3 \cdot 20 \\
1 \cdot 44\end{array}$ & 3.90 & $2 \cdot 86$ & $3 \cdot 28$ & 3.05 & $3 \cdot 78$ & & & \\
\hline
\end{tabular}

Diffusing CaPaCiTY.-The diffusing capacity usually decreased after the injection of clots but partially recovered before the next test in the following week (Table IV). In only one dog (dog 4) was there no reduction in diffusing capacity as a result of the embolization, and in some dogs reduction of over $50 \%$ was seen after the injection of 60 or $80 \mathrm{ml}$. of clot.

Lung Compliance.-The lung compliance usually decreased after the embolization (Table V). The decreased compliance usually occurred at the same time as the respiratory changes, that is, a few breaths after the rise in pulmonary artery pressure (Fig. 1), but in some cases a fall in compliance occurred without an accompanying change in the rate and depth of respiration. In many cases the respiration soon became slower and deeper, but the compliance only gradually increased towards its original value (Fig. 2).

Non-elastic Resistance.-The non-elastic resistance of the lungs was calculated from the intra-oesophageal pressure and tidal volume tracings in 10 dogs (Table VI). The non-elastic resistance after embolization was measured, if possible, when the compliance had decreased. When the compliance of the lungs decreased the non-elastic resistance usually increased, but in most dogs the changes were not large.

\section{Discussion}

The cause of the rapid, shallow breathing and of the rise in pulmonary artery pressure after pulmonary embolism is not known, neither is the cause of the decreased compliance.

The Rise in Pulmonary Artery Pressure.-There is an extensive literature on the effects of pulmonary embolism and the parts played by reflex action and by simple mechanical blockage of the pulmonary vessels. The small blood clots used in these experiments had passed through a tube of $1 \mathrm{~mm}$. diameter. Microscopic examination of the lungs showed clot only in vessels of $0.5 \mathrm{~mm}$. diameter and larger. Other investigators have usually found that reflex respiratory and cardiac effects are produced only by emboli of less than $100 \mu$ diameter (Weidner and Light, 1958). In the experiments reported here the pulmonary artery pressure rose but usually began to fall after about 20 to 30 seconds and these changes in pulmonary artery pressure were not accompanied by large changes in cardiac output; they were due to changes in resistance of the pulmonary vascular bed. The fall in resistance occurring about 20 to 30 seconds after the impaction of the embolus could be due to blood getting more easily past the clot, but this is unlikely to account for the large change in resistance which was seen in some cases and a more probable cause is relaxation of tone of the pulmonary vessels. This could be a relaxation of normal tone or a relaxation of spasm induced by the embolus either reflexly or by release of vasoactive substances. When mechanical obstruction of a pulmonary artery causes a rise in pulmonary artery pressure the pressure remains raised until the obstruction is released (Lategola and Rahn, 
1955). The fall in pulmonary artery pressure found in the present series is therefore unlikely to be due to relaxation of tone normally present in the pulmonary vessels but is much more likely to be due to relaxation of constriction resulting from impaction of the embolus. The rise in pulmonary artery pressure produced by embolization with plastic spheres of less than $100 \mu$ diameter could be prevented by complete denervation of the lung (Weidner and Light, 1958), suggesting that reflexes play a part in the pulmonary artery pressure rise. The rate of fall of the pulmonary artery pressure is too slow to be the result of stress relaxation of the pulmonary vessels (Sarnoff and Berglund, 1952). Megibow, Katz, and Steinitz (1942), using emboli of various sizes (seeds and starch granules), found a sustained rise in pulmonary artery pressure after embolization of dog lungs. The tachypnoea which accompanied the rise in pulmonary artery pressure also persisted. It might be thought that the transient rapid, shallow breathing and the peak of pulmonary artery pressure were due to the nature of the embolus or to some substance liberated from it, but Horres and Bernthal (1961), using small glass spheres, also reported only transient tachypnoea which in its time relationship resembles that found in the present experiment. Although the high pulmonary artery pressure found immediately after the injection of blood clot may have been due to vasoconstriction, either reflex or chemical, the short duration of this vasoconstriction makes it unlikely to be a factor in causing the death of the dogs.

An attempt to produce pulmonary hypertension by repeated doses of blood clot was made in only two of the dogs. One of these dogs died during the injection of clot and without a final measurement of pulmonary artery pressure being made. The other (dog 1) received $240 \mathrm{ml}$. of clot over a period of three months and was killed three months after the final injection. The pulmonary artery pressure at this time was normal. At necropsy the lungs appeared normal, and microscopic examination showed only one small artery which contained a mass of intimal fibrous tissue which had presumably developed by organization of clot. This finding is in agreement with that of Parker, Thomas, and Smith (Parker and Smith, 1958), who were unable to produce pulmonary hypertension in dogs by repeated injection of fibrin clot.

Rapid, Shallow Breathing.-The rapid, shallow breathing which occurs after pulmonary embolism is often considered to be secondary to changes in the pulmonary vessels or the circulation through the right heart (Megibow, Katz, and Feinstein, 1943 ; Whitteridge, 1950). In the present investigation rapid, shallow breathing occurred without any rise in the central venous pressure and it is unlikely to have been initiated by the vena caval reflex suggested by Megibow et al. (1943) but in all the cases in which rapid shallow breathing occurred the pulmonary artery pressure also rose. In some cases the first injection of clot caused rapid, shallow breathing and a rise in pulmonary artery pressure whilst subsequent injections caused no rapid, shallow breathing even though the pulmonary artery pressure rose still higher. Increase in the pulmonary artery pressure by blocking the pulmonary artery to one lung by a balloon (Lategola and Rahn, 1955) or by an encircling cuff (Moore and Binger, 1927) does not cause rapid, shallow breathing. Moore and Binger discussed the possible causes of the rapid, shallow breathing and concluded that it must be due to oedema of the lung. The compliance of the lung should be decreased when oedema occurs, either because of interstitial oedema or more probably because transudation of oedema fluid causes collapse of parts of the lung. In many of the dogs in this series the compliance fell at about the same time as the decrease in tidal volume, although the compliance change tended to occur later than the tidal volume change. The tidal volume often increased to normal by about one minute after reaching its minimum, but even when the tidal volume had recovered the compliance often remained low and increased slowly later. In spite of this dissociation between changes in tidal volume and compliance, in almost all cases when the tidal volume fell after embolization the compliance was also decreased.

In some pathological conditions a decreased compliance of the lungs may be the cause of rapid, shallow breathing (Marshall, McIlroy, and Christie, 1954 ; Marshall and Christie, 1954) since the rate and depth of breathing seem to be so adjusted that minimal respiratory work is performed, but there is no evidence that the decreased compliance was responsible for the rapid, shallow breathing in these dogs. When the breathing of these dogs was shallow the intra-oesophageal pressure swing was less than normal, and this reduction would not occur if the rate change was due to a change in the optimal frequency. During the period of rapid, shallow breathing the alveolar ventilation is often less than normal, indicating a disturbance of the control of respiration. When the total elastic resistance to breathing is increased 
by having a dog breathe from a closed vessel the intra-oesophageal pressure swing gradually increases and the respiratory rate does not increase appreciably (unpublished observations).

Weidner and Light (1958) have shown that both the tachypnoea and the rise of pulmonary artery pressure which occur after embolization with small plastic spheres can be prevented by complete denervation of the lung. Although Torrance and Whitteridge (1948) found that the tachypnoea due to starch embolization was still present after cooling the vagi to $6^{\circ} \mathrm{C}$., vagotomy is often stated to prevent reflex rapid, shallow breathing (Weidner and Light, 1958; Horres and Bernthal, 1961; Megibow et al., 1943 ; Holden, Shaw, Cameron, Shea, and Davis, 1949). Vagotomy produces a very abnormal type of breathing which one would not expect to change into the rapid, shallow breathing normally seen after embolization. During this series of experiments small blood clots were also put into two dogs after bilateral cervical vagotomy. In one dog the resting respiratory rate was 5 per minute and the tidal volume $600 \mathrm{ml}$.; for a few breaths after the embolism the tidal volume fell to $500 \mathrm{ml}$., and the rate rose to 7 per minute. In the other dog the initial respiratory rate was 10 per minute, the tidal volume $200 \mathrm{ml}$., and no Hering-Breuer reflex could be elicited. After the embolism the tidal volume fell to $50 \mathrm{ml}$. and the rate rose to 27 per minute.

Complance.-The compliance of the lungs often decreased after embolization. When compliance fell there was always a rise in pulmonary artery pressure, but a rise in pulmonary artery pressure was not always accompanied by a fall in compliance, and in some dogs subsequent doses of clots caused a large rise in pulmonary artery pressure but a small change in compliance. The change in compliance could not be related to any particular level of pulmonary artery pressure. The decrease in compliance often occurred much later than the maximum pulmonary artery pressure, and it is tempting to suppose that transudation of fluid into the lungs during the period of

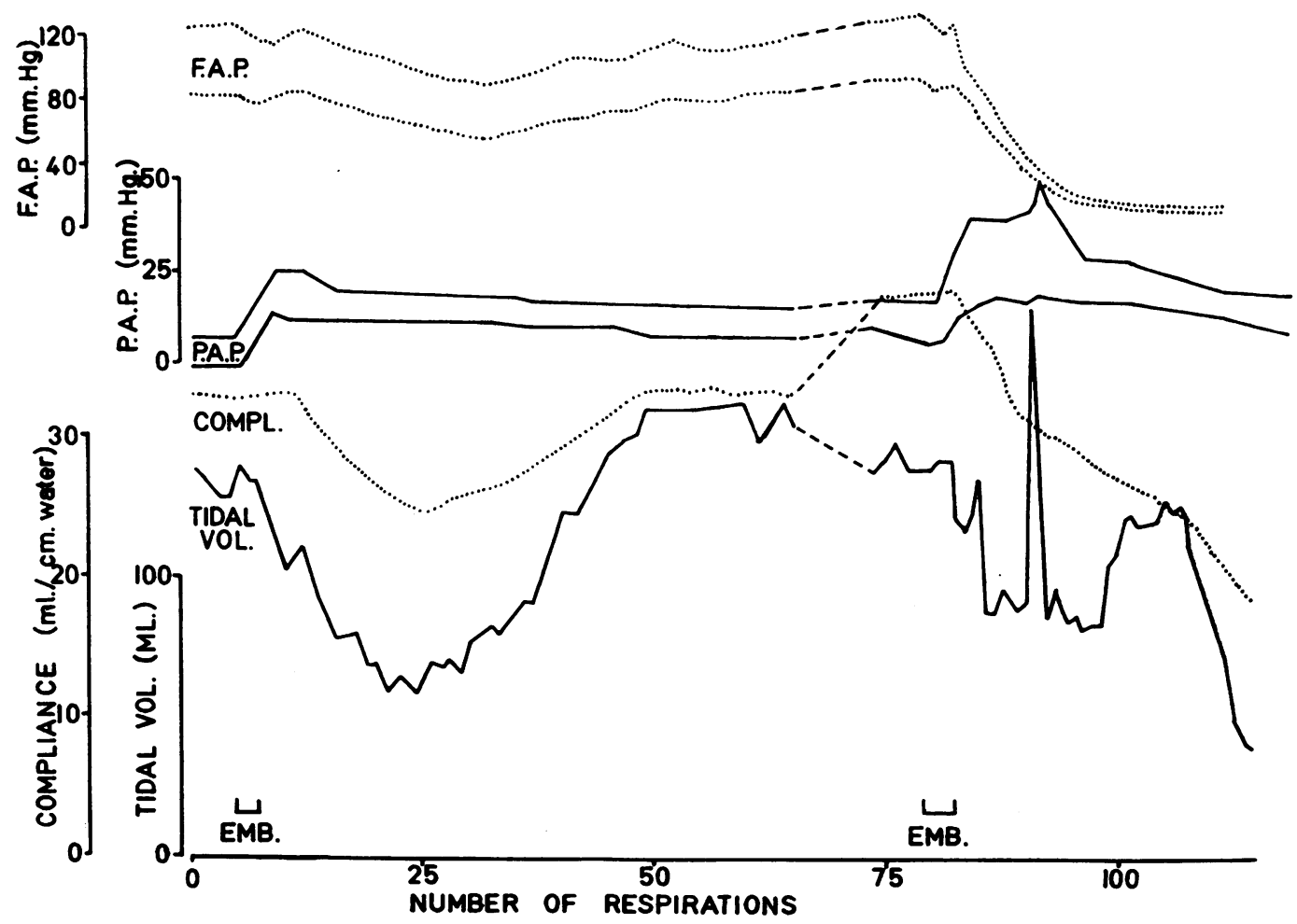

FIG. 3. The effect on respiration and haemodynamics of the intravenous injection of small blood clot (dog 4, 6/7) : F.A.P.=Femoral artery pressure; P.A.P.= pulmonary artery pressure; the time scale is shown as "number of respirations." 
high pressure increased the stiffness of the lungs ; but in some cases the compliance decreased even though the pulmonary artery pressure was not high enough to cause transudation. It is possible that other factors, such as bronchial narrowing in the embolized part of the lung (Severinghaus et al., 1961), may cause local collapse of lung tissue and decrease the compliance. Although the decrease in compliance was not synchronous with the rap:d, shallow breathing, it appeared possible that the small tidal volume might cause an apparent change in compliance. Two of the dogs were ventilated passively in a body plethysmograph to produce rapid, shallow breathing. The compliance measured during the period of rapid, shallow breathing was not significantly different from that measured during breathing of normal depth. Cahill, Attinger, and Byrne (1961) for the same reason ventilated dogs at a constant rate and depth during embolization and still found a decrease in compliance as a result of embolization by barium sulphate. Cahill et al. also found a significant increase in non-elastic resistance of the lungs. The haemodynamic changes and the type of respiration after barium sulphate embolization were different from those seen after embolization by blood clot.

Although compliance has been measured throughout this study by means of oesophageal pressure measurements with their attendant disadvantages (Mead and Gaensler, 1959 ; Knowles, Hong, and Rahn, 1959), there is no reason to believe that artefacts of pressure measurement could have caused any of the changes of compliance which were measured. Bondurant, Mead, and Cook (1960) have suggested that changes in cardiac distension may affect the size of the intraoesophageal pressure swings during respiration, but in the dogs in this series changes in pulmonary vascular pressure, which might have caused changes in heart distension, were often not accompanied by changes in compliance and the compliance sometimes decreased when the pulmonary vascular pressures were returning to normal.

The Cause of Death.-The cause of death in those dogs which died soon after the addition of clots appeared to be right heart failure due to mechanical obstruction to the circulation. In some instances respiration suddenly became more rapid and shallow and then stopped (Fig. 3). In some the respiration, after being rapid and shallow, had begun to increase in depth when it suddenly stopped, and in others there was no change in rate or depth of respiration until the terminal apnoea. The apnoea appeared to be caused by a fall in the systemic and pulmonary artery pressure and the heart often continued to beat for several minutes after respiration had ceased.

\section{SUMMARY}

The respiratory and haemodynamic effects of embolization by small blood clots were studied in 10 anaesthetized dogs. Injection of $20 \mathrm{ml}$. of finely divided blood clot usually caused a rise in pulmonary artery pressure and transient, rapid, shallow breathing. Sixty or $80 \mathrm{ml}$. of clot, given in divided doses on one day, was not usually fatal, but 40 or $60 \mathrm{ml}$. given one week later usually caused death.

The injection of clots often caused a fall in compliance of the lung and a rise in non-elastic resistance. Rapid, shallow breathing was usually accompanied by a fall in compliance, but the fall in compliance did not appear to cause the rapid, shallow breathing.

Pulmonary vascular resistance increased transiently after injection of clot; the cause of this is discussed.

The diffusing capacity of the lungs for carbon monoxide decreased as a result of the embolization, but no evidence for right-to-left shunt of blood was found in these experiments.

\section{REFERENCES}

Allison, P. R., Dunnill, M. S., and Marshall, R. (1960). Thorax, 15, 273.

Bondurant, S., Mead, J., and Cook, C. D. (1960). J. appl. Physiol., 15,875 .

Cahill, J. M., Attinger, E. O., and Byrne, J. J. (1961). Ibid., 16, 469. Hackney, J. D., Sears, C. H., and Collier, C. R. (1958). Ibid., 12, 425 Holden, W. D., Shaw, B. W., Cameron, D. B., Shea, P.' J., and Davis, J. H. (1949). Surg. Gynec. Obstet., 88, 23.

Horres, A. D., and Bernthal, T. (1961). J. appl. Phvsiol, 16, 842.

Knowles, J. H., Hong, S. K., and Rahn, H. (1959). Ibid., 14, 525.

Lategola, M., and Rahn, H. (1955). WADC Tech. Rep., 55-357, p. 180 .

Marshall, R., and Christie, R. V. (1954). Clin. Sci., 13, 403.

Mcilroy, M. B. and Christie, R. V. (1954). Iibid., 13, 137.

Mead, J., and Gaensier, E. A. (1959). J. appl. Phvsiol., 14, 81.

Megibow, R. S., Katz, L. N., and Feinstein, M. (1943). Arch. intern. Med., 71, 536.

and Steinitz, F. S. (1942). Surgery, 11, 19.

Moore, R. L., and Binger. C. A. L. (1927). J. exp. Med., 45, 655.

Neergaard, K. von, and Wirz, K. (1927). Z. klin. Med., 105, 35.

Parker, B. M., and S.nith, J. R. (1958). Amer. J. Med., $24,402$.

Sarnoff, S. J., and Berglund, E. (1952). Amer. J. Phvsiol., 171, 238.

Severinghaus. J. W. Swenson, E. W. Finley, T. N., Lategola, M. T. and Williams, J. (1961). J. appl. Phusiol., 16, 53.

Torrance, R. W., and Whitteridge, D. (1948). J. Physiol. (Lond.), $107,6 P$.

Weidner, M. G., and Light, R. A. (1958). Ann. Surg., 147, 895.

Whitteridge, D. (1950). Physiol. Rev., 30, 475. 